\title{
Megakaryocytes in pleural and peritoneal fluids: prevalence, significance, morphology, and cytohistological correlation
}

\author{
NB KUMAR AND B NAYLOR \\ From the Department of Pathology, The University of Michigan, Ann Arbor, Michigan, 48109, USA
}

SUMMARY Over a period of 22 years, 4844 pleural and peritoneal fluids from 3279 patients were examined cytologically. Megakaryocytes were found in the fluids from five patients. The clinical diagnoses in the five patients were agnogenic myeloid metaplasia, chronic myeloid leukaemia, and lymphocytic lymphoma. All of these patients had persistent serous effusions. Megakaryocytes in serous fluids occurred in three forms: (1) a large type with abundant cytoplasm and multilobed nuclei, (2) a smaller type with a high nucleocytoplasmic ratio and unlobed nuclei, and (3) anucleate cytoplasmic masses. Foci of agnogenic myeloid metaplasia found on the serous surfaces at necropsy of two patients contained megakaryocytes similar to those in the corresponding effusions. The clinical course of our patients confirmed that the presence of megakaryocytes in serous fluids signifies an advanced haematopoietic malignancy.

Megakaryocytes, normal inhabitants of bone marrow, are occasionally seen in peripheral blood and on a rare occasion they may be found in sputum ${ }^{1}$ due to the passage of circulating megakaryocytes through the pulmonary alveolar walls. They have also been described in cerebrospinal fluid due to unintentional puncture of a vertebra during lumbar puncture. ${ }^{2}$

Megakaryocytes have also been described in pleural and peritoneal fluids ${ }^{1-4}$ in association with myeloproliferative disorders, presumably due to the development of extramedullary haematopoiesis on the pleura or peritoneum. Such foci of haematopoiesis may develop in various organs whenever the haematopoietic activity of the bone marrow is inadequate to meet normal requirements. This compensatory metaplastic phenomenon occurs, for example, when the bone marrow is diffusely replaced by neoplasm such as lymphoma. On the other hand, extramedullary haematopoiesis may be a truly neoplastic change as in agnogenic myeloid metaplasia (AMM). In addition to the extramedullary haematopoiesis, AMM is also characterised by myelofibrosis and the proliferation of atypical megakaryocytes.

The finding of megakaryocytes in pleural and peri-

Received for publication 8 April 1980 toneal fluids has, in our experience, been a rare event. This paper concerns the prevalence, significance, and morphological features of megakaryocytes in pleural and peritoneal fluids examined in our laboratory and in two cases correlates the cytological findings with the findings at necropsy.

\section{Material and methods}

The records of the Cytopathology Laboratory in the Department of Pathology of The University of Michigan from 1957 to 1979 were searched for specimens of pleural, peritoneal, and pericardial fluids that contained megakaryocytes.

The sediment obtained by centrifugation of the fluids was smeared, fixed in $95 \%$ ethanol, and stained by the Papanicolaou method. In four of the five cases the sediment was clotted, and this, with any spontaneously occurring clot, was fixed in formalin, embedded in paraffin, sectioned, and stained with haematoxylin and eosin. Wright-stained specimens of peritoneal fluid prepared in the Cytospin were available from patients 1 and 2 .

The original histological sections of biopsy material were reviewed and new sections were prepared when necessary. Tissues from the two patients who came to necropsy were embedded in paraffin, 1153 
Table 1 Megakaryocytes in pleural and peritoneal fluids: clinical and pathological features

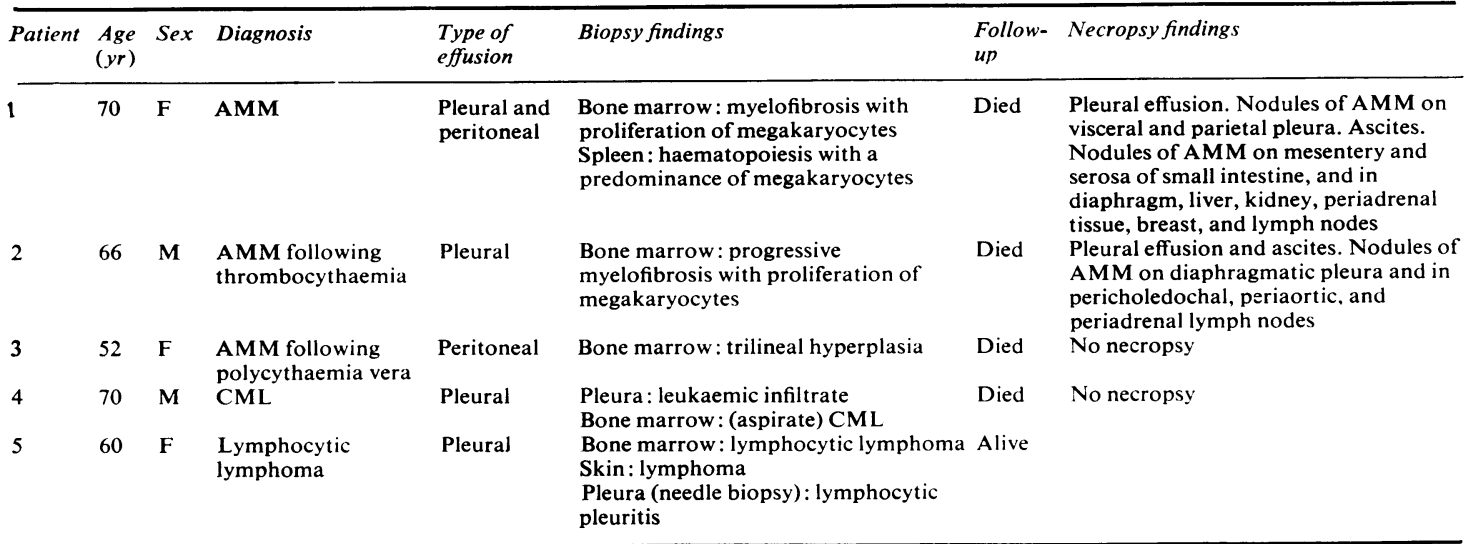

sectioned, and stained with haematoxylin and eosin.

The overall cellularity of each smear was subjectively graded from + to +++ . In addition, the number of megakaryocytes on each smear was counted. Fewer than five per slide was graded as + , six to 10 per slide as ++ , and more than 10 per slide as +++ .

The clinical information in the patients' medical records was also reviewed.

\section{Results}

\section{PREVALENCE OF MEGAKARYOCYTES}

During 22 years, 4844 pleural and peritoneal fluids from 3279 patients were examined and megakaryocytes were found in such fluids from only five patients.

\section{CLINICAL FINDINGS}

A summary of the major clinical features for each patient is presented in Table 1. Three of the five patients were women and two were men, all within an age range of 52-70 years. Three of the five patients had AMM, one had chronic myeloid leukaemia (CML), and one had lymphocytic lymphoma. Each patient had a persistent serous effusion. Patient 1, with a diagnosis of $\mathrm{AMM}$, had recurrent massive pleural effusions for one year before death. Patient 2 with a diagnosis of $\mathrm{AMM}$, had recurring massive pleural effusions for two years before he died and developed ascites one week before death. Patient 3 developed AMM after a 13-year history of polycythaemia vera. She had recurring ascites during the three years before she died. The duration of pleural effusion in patient 4 , who had CML, was not available from the medical records. He died two months after admission to our hospital. Patient 5 , who has lymphocytic lymphoma and is still alive, has had recurrent bilateral pleural effusions for the last three years.

CYTOLOGICAL FINDINGS

The cytological features of the pleural and peritoneal fluids from the five patients are summarised in Table 2. The cellular components of the sediments consisted of various proportions of mesothelial cells, histiocytes, erythrocytes, and megakaryocytes. The fluids from three of the five patients also contained neoplastic cells in the form of immature granulocytes (patients 1 and 4) or lymphoma cells (patient 5).

The number of megakaryocytes in the smears ranged from + to +++ . They were not adherent to each other. In the Papanicolaou-stained smears, the diameters of the megakaryocytes ranged from 20 to

Table 2 Megakaryocytes in pleural and peritoneal fluid: cytological findings

\begin{tabular}{|c|c|c|c|c|}
\hline Patient & Character of effusions & Cellularity & Background cells & No. of megakaryocytes \\
\hline 1 & $\begin{array}{l}\text { Cloudy yellow to } \\
\text { bloody }\end{array}$ & $\begin{array}{l}\text { Pleural: }+ \text { to }++ \\
\text { Peritoneal: }++\end{array}$ & $\begin{array}{l}\text { Immature granulocytes } \\
\text { Histiocytes and mesothelial cells }\end{array}$ & +1 \\
\hline 2 & $\begin{array}{l}\text { Cloudy yellow to } \\
\text { bloody }\end{array}$ & $\begin{array}{l}\text { Pleural: }++ \\
\text { Peritoneal (necropsy) }\end{array}$ & $\begin{array}{l}\text { Immature granulocytes, erythrocytes, histiocytes, mesothelial } \\
\text { cells } \\
\text { Mesothelial cells }\end{array}$ & + to $+i$ \\
\hline 3 & Cloudy light red & Peritoneal: + & Erythrocytes and leukocytes & + \\
\hline $\begin{array}{l}4 \\
5\end{array}$ & $\begin{array}{l}\text { Cloudy orange to red } \\
\text { Cloudy yellow }\end{array}$ & $\begin{array}{l}\text { Pleural: }++ \text { to } t++ \\
\text { Pleural: } t+\text { to } t++\end{array}$ & $\begin{array}{l}\text { Leukaemic cells and erythrocytes } \\
\text { Lymphoma cells, occasional histiocytes }\end{array}$ & $\begin{array}{l}+\cdots \text { to }+:= \\
+\div\end{array}$ \\
\hline
\end{tabular}




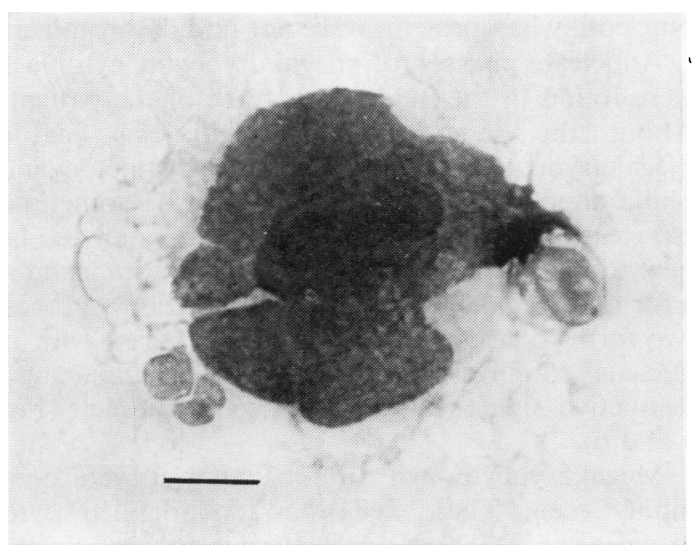

Fig. 1 Patient 1. Cytospin centrifuge preparation of peritoneal fluid. A megakaryocyte with a multilobed nucleus with lobes superimposed on each other. The cell has a large amount of irregularly outlined cytoplasm. (Wright stain. Mark is $10 \mu \mathrm{m}$.)

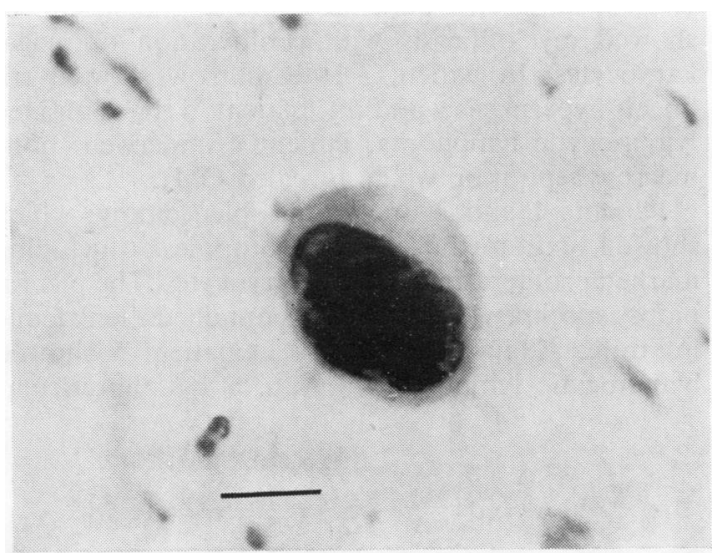

Fig. 2 Patient 5. Smear of pleural fluid. A megakaryocyte with a multilobed nucleus. The superimposed lobes of the nucleus accentuate the hyperchromasia. (Papanicolaou stain. Mark is $15 \mu \mathrm{m}$. )

80 microns. Their shapes were round to ovoid. Occasional megakaryocytes were fusiform or irregular in shape, which may have been an artifact created by the smearing of the sediment. Most of the megakaryocytes had abundant cytoplasm. With the Papanicolaou stain the cytoplasm was either acidophilic, cyanophilic, or amphophilic. In some megakaryocytes the cytoplasm was finely granular, a feature that was better seen in the Wright-stained preparations. Essentially, three basic forms of megakaryocytes were present: (1) megakaryocytes

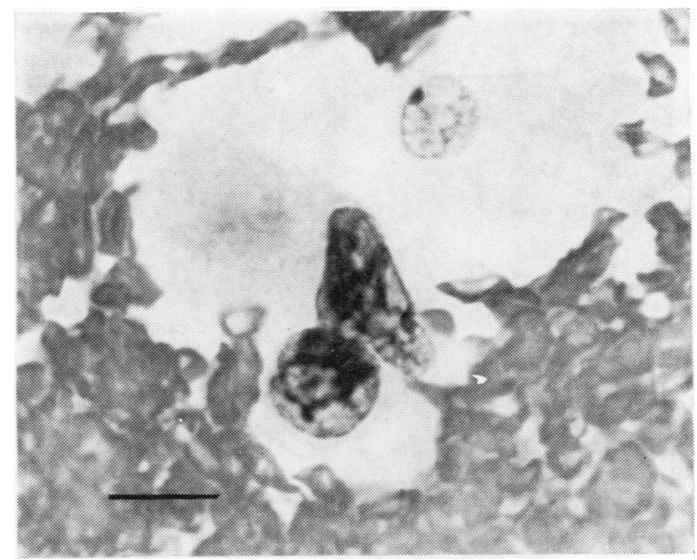

Fig. 3 Patient 2. Smear of pleural fluid. A megakaryocyte with an eccentric bilobed nucleus. The chromatin is coarsely clumped, leaving a clear area between the clumped chromatin and the nuclear membrane. The nuclear membrane is indented and appears to be delicate. (Papanicolaou stain. Mark is $15 \mu \mathrm{m}$.)

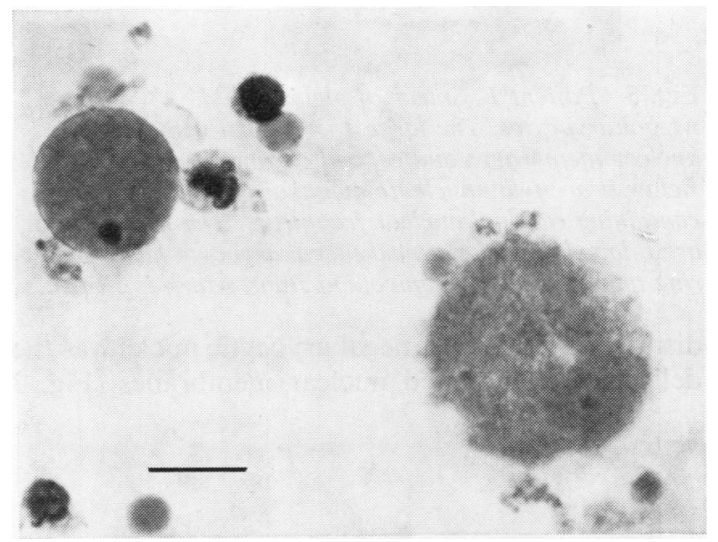

Fig. 4 Patient 1. Smear of peritoneal fluid. Two anucleate megakaryocytes. Both contain residual nuclear material. (Papanicolaou stain. Mark is $19 \mu \mathrm{m}$.)

with multilobed nuclei and a large amount of cytoplasm, (2) anucleate cytoplasmic masses, and (3) megakaryocytes with unlobed nuclei and a small amount of cytoplasm.

The multilobed megakaryocytes contained nuclei with two or more lobes that were frequently superimposed, thereby accentuating their hyperchromasia (Figs 1 and 2). Frequently, the chromatin was irregularly clumped, generally toward the centre of the nucleus, leaving a clear area between the clumped chromatin and the nuclear membrane (Fig. 3). A 


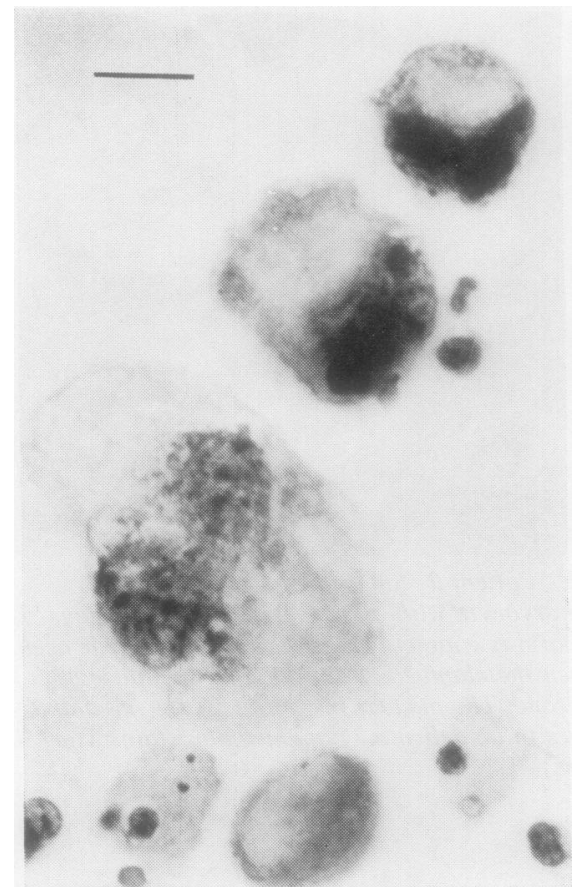

Fig. 5 Patient 1. Smear of pleural fluid.

Megakaryocytes. The largest is bilobed with delicate nuclear membranes and central chromatin clumping.

Below it are two anucleate megakaryocytic masses, one containing residual nuclear fragments. The two above it are bilobed. The uppermost megakaryocyte has a signetring appearance. (Papanicolaou stain. Mark is $19 \mu \mathrm{m}$.)

distinctive feature of megakaryocytic nuclei was their delicate and indented nuclear membranes (Fig. 3).

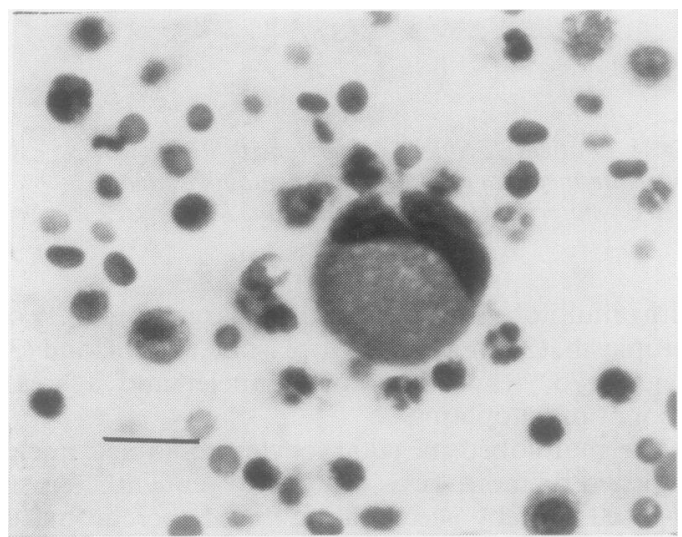

Fig. 6 Patient 5. Cell block of pleural fluid. A megakaryocyte with an eccentric multilobed nucleus, imparting a signet-ring appearance to the cell. (Haematoxylin and eosin. Mark is $15 \mu \mathrm{m}$.)
Nucleoli, when present, were not unduly prominent. Anucleate masses of megakaryocytic cytoplasm were found in the fluids from three of the patients. About the same size and shape as mature megakaryocytes, they appeared as distinct cyanophilic anucleate masses (Figs 4 and 5). Sometimes bare nuclei, apparently from megakaryocytes, lay next to these anucleate masses. What we interpret as a form of megakaryocyte intermediate between these two forms consisted of a cytoplasmic mass with its nucleus 'compressed' at the periphery, imparting a distinctive signet-ring appearance to the cell (Figs 5 and 6).

Megakaryocytes with unlobed nuclei were commonly seen. These cells had well-defined cytoplasmic borders, high nucleocytoplasmic ratios, and a more uniform chromatin pattern than the megakaryocytes with lobed nuclei (Fig. 7). These megakaryocytes with unlobed nuclei may represent less mature forms.

\section{BIOPSY FINDINGS}

Bone marrow biopsies were performed on patients $1,2,3$, and 5 . In patients 1 and 2 , the specimens showed myelofibrosis with proliferation of megakaryocytes. In patient 3, the marrow showed trilineal hyperplasia, and in patient 5 it contained lymphocytic lymphoma. Patient 4 underwent bone marrow aspiration which revealed CML.

Patients 1 and 2 underwent splenectomy, which showed extramedullary haematopoiesis including marked proliferation of megakaryocytes. The pleural biopsy specimen from patient 4 contained a leukaemic infiltrate. Additional biopsies in patient 5 showed lymphocytic lymphoma in skin, a lymphoreticular

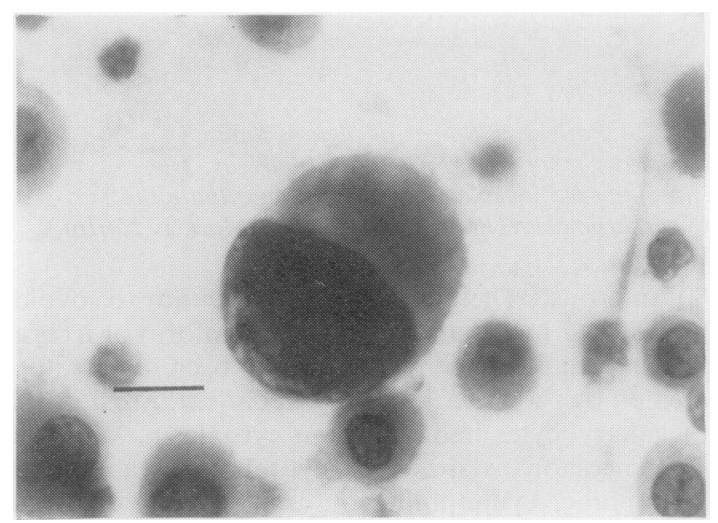

Fig. 7 Patient 2. Smear of pleural fluid. A megakaryocyte with an unlobed nucleus. (Papanicolaou stain. Mark is $15 \mu \mathrm{m}$.) 


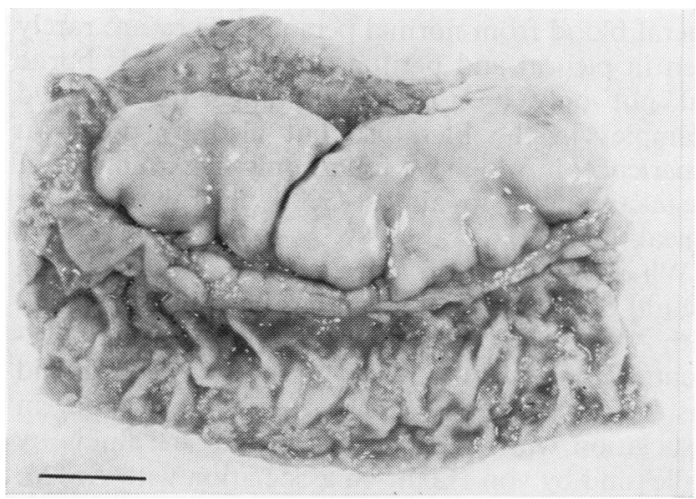

Fig. 8 Patient 1. Small intestine. Confluent nodules of AMM in the mesentery. (Mark is $12 \mathrm{~mm}$.)

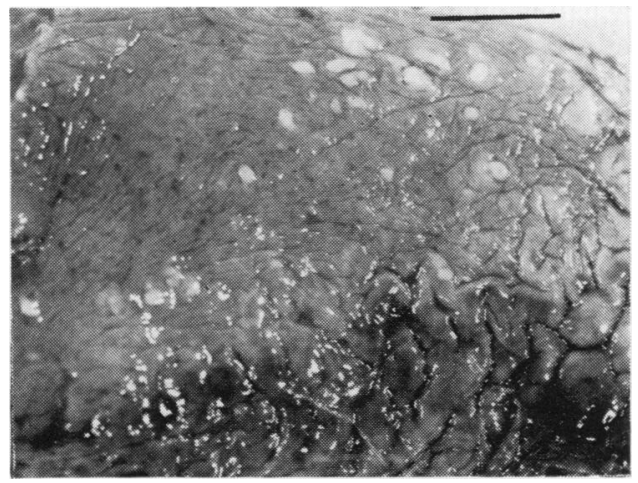

Fig. 9 Patient 1. Lung. Slightly raised nodules of extramedullary haematopoiesis on the visceral pleura. (Mark is $7.7 \mathrm{~mm}$.) infiltrate in the liver, and lymphocytic pleuritis.

\section{NECROPSY FINDINGS}

Patient 1 had massive ascites $(5300 \mathrm{ml}$ of serosanguineous fluid) and a right pleural effusion. The omentum and mesentery contained masses of greywhite, firm tissue (Fig. 8). Similar masses were found in peripancreatic and periadrenal soft tissues. The liver weighed $3140 \mathrm{~g}$ and contained numerous greywhite nodules. Smaller nodules were found on the visceral pleura (Fig. 9) and in the kidneys, and a mass $12 \mathrm{~cm}$ in diameter invaded the left hemidiaphragm. The bone marrow was dry, grey-white, and sclerotic.

Microscopical examination revealed that the nodules were composed of fibrous connective tissue mingled with haematopoietic cells, including atypical and bizarre megakaryocytes (Fig. 10). Such foci of haematopoiesis were found in lymph nodes, visceral and diaphragmatic pleura, peritoneum, adrenal glands, peripancreatic and periadrenal adipose tissue, kidney, liver, gallbladder, pulmonary parenchyma, heart, breast, and stomach. The bone marrow was fibrotic and contained scattered enlarged and atypical megakaryocytes in addition to other haematopoietic cells. Each of the three morphological types of megakaryocyte seen in the cytological preparations was readily identified in the histological sections of the nodules, bone marrow, and lymph nodes.

The left and right pleural cavities of patient 2 contained $2700 \mathrm{ml}$ and $800 \mathrm{ml}$ of serosanguineous fluid, respectively, and the peritoneal cavity contained $2000 \mathrm{ml}$ of serous fluid. Other pertinent gross findings were a granular appearance of the pleural

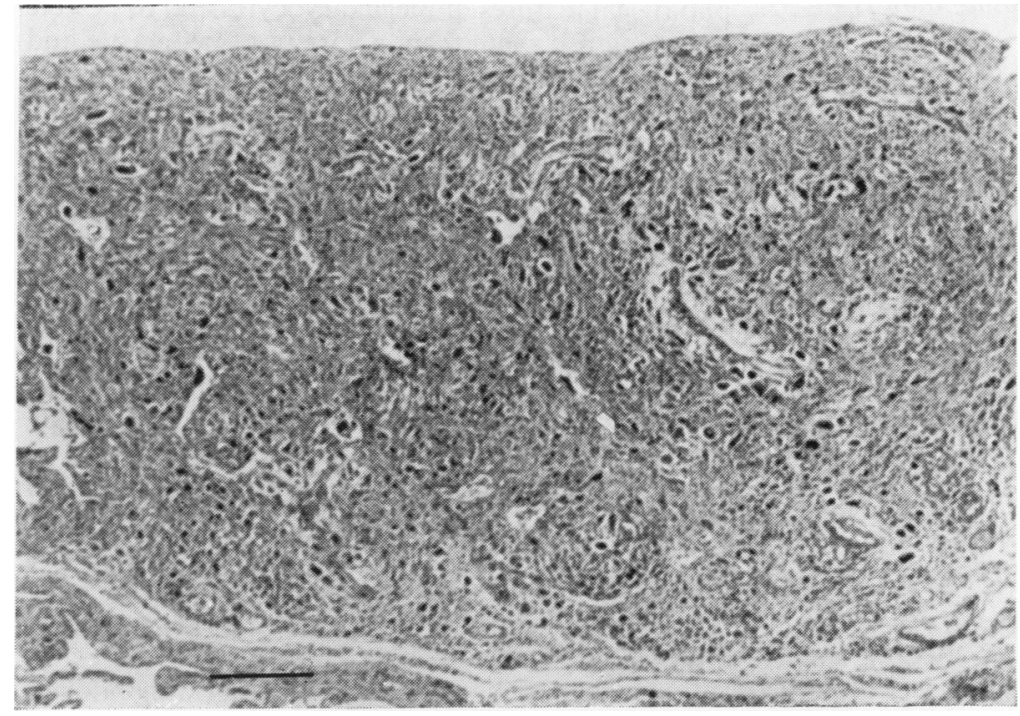

Fig. 10 Surface of lung. Nodule of AMM consisting of fibrous tissue containing haematopoietic cells, with a predominance of megakaryocytes. (Haematoxylin and eosin. Mark is $300 \mu \mathrm{m}$.) 


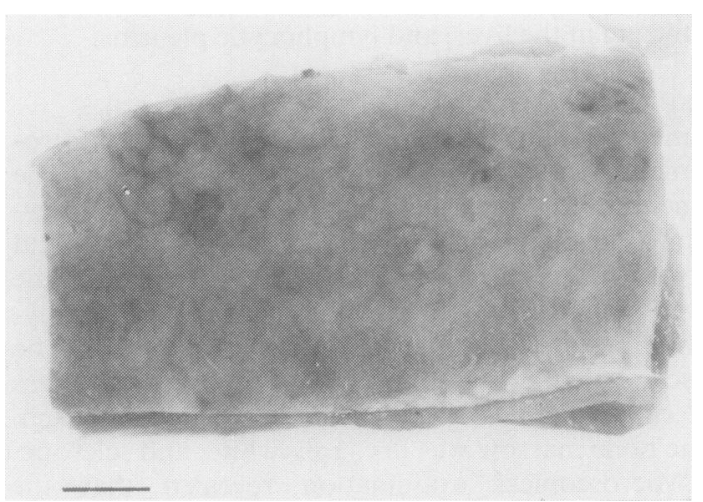

Fig. 11 Patient 2. Diaphragm. Pleural surface showing irregularly shaped plaques of AMM. (Mark is $3.6 \mathrm{~mm}$.)

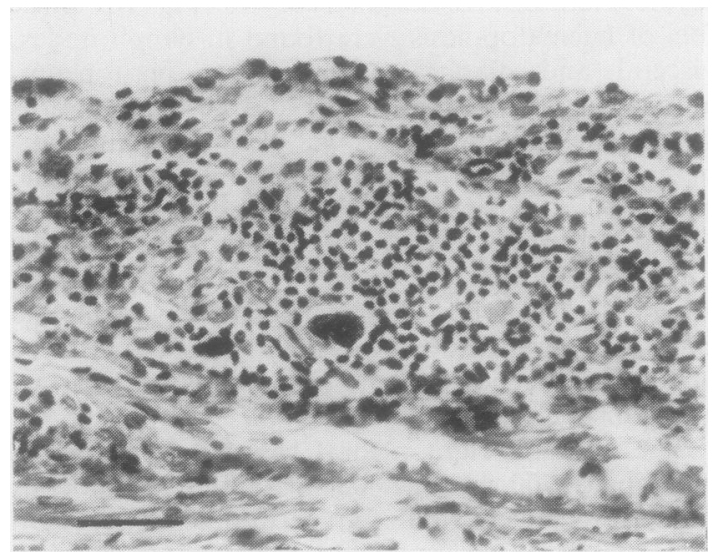

Fig. 12 Patient 2. Diaphragmatic pleura.

Extramedullary haematopoiesis including megakaryocytes. (Haematoxylin and eosin. Mark is 48 нm.)

surface of the diaphragm (Fig. 11), enlarged lymph nodes in the porta hepatis, patchy pneumonic consolidation of the lungs, and sclerotic bone marrow.

Microscopically, foci of haematopoiesis with prominent atypical megakaryocytes were seen in the pulmonary parenchyma, lymph nodes, kidney, liver, periadrenal and periaortic tissues, and on the pleural surface of the diaphragm (Fig. 12). The bone marrow was fibrotic and contained numerous atypical megakaryocytes.

\section{Discussion}

Megakaryocytes are rarely found in routinely prepared smears of peripheral blood in normal states, although they can be found in very small numbers in smears of white cell concentrates of peri- pheral blood from normal persons. ${ }^{5}$ They are rarely seen in pleural and peritoneal fluids. This is borne out not only by the small number of reported examples in the literature but also by our own experience, in which we found only five examples of megakaryocytes in almost 5000 pleural and peritoneal fluids.

Whenever megakaryocytes have been seen in pleural or peritoneal fluids it has been in association with AMM or leukaemia or lymphomatous involvement of bone marrow. Their presence in pleural fluid has been reported by Spriggs and Boddington ${ }^{2}$ in association with CML, and in peritoneal fluids by Calle $^{3}$ and by von Haam ${ }^{4}$ in association with AMM. Similarly, our patients all had some form of myeloproliferative disorder or neoplastic involvement of the bone marrow. Three had AMM, one had CML, and one had lymphocytic lymphoma.

Since grossly visible nodules of AMM may resemble metastatic neoplasm, ${ }^{6}$ it is important not to misinterpret atypical megakaryocytes as malignant mesenchymal or epithelial cells in histological sections of such nodules or in any associated serous effusion. They have been mistaken for malignant cells in peripheral blood from patients known to have cancer, and numerous reports have appeared regarding the difference between megakaryocytes and malignant cells in peripheral blood. ${ }^{7} 8$ Furthermore, since AMM could manifest itself by tumour formation, as exhibited in our case 1 and as previously described, ${ }^{6-11}$ it is conceivable that one may be presented with a cellular sample obtained by aspirating a nodule of AMM or a lymph node involved with AMM. In such a situation, it would be extremely important to be familiar with the morphological features of megakaryocytes and to be able to distinguish them from a variety of neoplastic and non-neoplastic cells.

Generally, it should not be difficult to identify mature megakaryocytes in a serous fluid. Many of them are outstandingly large, much larger than typical carcinoma cells or cells of lymphomas, and they do not adhere to each other as do carcinoma cells. Those megakaryocytes which have abundant cytoplasm do not exhibit the marked vacuolisation that is frequently seen in adenocarcinoma cells. Mature megakaryocytes contain multilobed nuclei with nuclear membranes that are not unduly prominent. In contrast, the nuclei of carcinoma cells may be multiple but they are not usually lobed. In addition, the nuclei of carcinoma cells are usually angulated and have sharp, heavy membranes. The nucleoli of carcinoma cells are frequently very prominent, whereas the nucleoli of megakaryocytes are usually small and may be difficult to perceive. Those megakaryocytes with unlobed or only bilobed 
nuclei and megakaryocytes with degenerated pyknotic nuclei are, perhaps, the ones that are more likely to be confused with malignant cells. Reed-Sternberg cells could be confused with megakaryocytes. However, they are generally smaller than megakaryocytes and have a high nucleocytoplasmic ratio, welldefined, heavy nuclear borders, and prominent nucleoli. Benign multinucleated mesothelial cells bear some resemblance to megakaryocytes although, unlike megakaryocytes, they contain distinctly multiple rather than lobed nuclei, which are smoothly round to ovoid with sharply defined borders. In addition, mesothelial cells frequently adhere to each other.

The explanation for the presence of megakaryocytes in serous fluids was obvious in two of our five patients: nodules of AMM on the surface of the pleura or peritoneum and in various other abdominal organs. Although we are not aware of any reports which demonstrated such a histocytological correlation, the existence of this situation was hypothesised by Calle. ${ }^{3}$ The presence of megakaryocytes in the peritoneal fluid of patient 3, who had AMM, was most likely due to nodules of AMM on the peritoneum. In patient 4 , diagnosed as having CML, AMM was also considered in the differential diagnosis, and, therefore, the source of the megakaryocytes in his pleural fluid may have been pleural foci of AMM.

In patient 5, who has lymphocytic lymphoma, there are two likely explanations for the presence of megakaryocytes in her pleural fluid. The more likely is the presence of compensatory extramedullary haematopoiesis on the pleura, although it is possible that an increased number of circulating megakaryocytes in peripheral blood, which is known to occur in the presence of various malignant neoplasms, may account for the presence of the megakaryocytes in the pleural fluid.

Finally, the question whether any blood in the serous fluids contributed to the presence of the megakaryocytes should be considered. Against this, the number of megakaryocytes in different specimens of serous fluid from the same patient did not increase with increasing bloodiness of the specimen.
The finding of megakaryocytes in a serous fluid should be reported because it may be a manifestation of some type of haematopoietic malignancy. Their presence seems to denote an advanced serious disorder and may be an ominous sign as four of our five patients died within four to 90 days after the finding of megakaryocytes in the serous effusions.

We gratefully acknowledge the assistance of Patricia M Novak, who took the photomicrographs.

\section{References}

${ }^{1}$ Koss LG. Diagnostic Cytology and Its Histopathologic Bases. 3rd ed. Philadelphia: JB Lippincott Company, 1979.

2 Spriggs AI, Boddington MM. The Cytology of Effusions and of Cerebrospinal Fluid. 2nd ed. London: William Heinemann Medical Books, 1968.

${ }^{3}$ Calle S. Megakaryocytes in an abdominal fluid. Acta Cytol $1968 ; 12: 78-80$.

${ }^{4}$ von Haam E. Cytology of transudates and exudates. Monographs in Clinical Cytology. Basel, New York: S Karger, 1977.

${ }^{5}$ Alexander RF, Spriggs AI. The differential diagnosis of tumour cells in circulating blood. J Clin Pathol 1960;13: 414-24.

- Estenez JM, Enrique EG, Moran TJ. Acute megakaryocytic myelofibrosis. Case report of an unusual myeloproliferative syndrome. Am J Clin Pathol 1974;62:52-9.

${ }^{7}$ Kierszenbaum AL, Tres LL. Differential diagnosis of megakaryocytes from cancer cells in peripheral blood. Acta Cytol 1964;8:91-4.

8 McGrew EA, Romsdahl MM, Valaitas J. Differentiation of hematopoietic elements from tumor cells in blood. Acta Cytol 1962;6:551-4.

${ }^{9}$ Glew RH, Haese WH, McIntyre PR. Myeloid metaplasia with myelofibrosis: The clinical spectrum of extramedullary hematopoiesis and tumor formation. Johns Hopkins Med J 1973;132:253-70.

${ }^{10}$ Leiberman PH, Rosnoli RV, Ley AB. Extramedullary myeloid tumors in primary myelofibrosis. Cancer 1965; 18:727-36.

11 French HE, Yates DR. Myelofibrosis and myeloid tumors as a surgical disease. South Med J 1970;63:387-91.

Requests for reprints to: Dr NB Kumar, Box 45, University of Michigan Medical Center, Ann Arbor, Michigan, 48109, USA. 